\title{
Defining piRNA expression
}

SMALL RNAS

PIWI-interacting RNAs (piRNAs) are short, 21-35-nucleotide long single-stranded RNAs with roles in germline transposon silencing and gametogenesis. Although much progress has been made in characterizing piRNA-processing mechanisms and effector functions, how piRNA-encoding loci are recognized and regulated by the transcriptional machinery is poorly characterized. Two recent studies provide new insights from diverse species.

piRNA-encoding loci in most species - such as flies and mammals - are transcribed as long, multikilobase precursor transcripts that are processed into various piRNAs. To gain insight into how mammalian piRNA-encoding loci might be regulated, Li et al. carried out high-throughput sequencing of total RNA and processed piRNAs from six developmental stages of mouse testes and mapped these reads to the corresponding genomic loci.

The authors were particularly interested in investigating the regulatory mechanisms of the burst piRNAs spermatogenesis. They identified $\sim 100$ genomic loci from which piRNA production typically increases 6,000-fold during pachytene. Analysing the sequence features at these genes, they frequently found a putative binding site for the MYB family of transcription factors near the transcription start site. As the expression of AMYB (also known as MYBL1) mirrored that of pachytene piRNAs during spermatogenesis, the authors tested whether this could be the key MYB family member that regulates the transcription of pachytene piRNA precursors. Chromatin immunoprecipitation experiments confirmed AMYB binding near the transcription start sites of pachytene piRNA genes, and Amyb-mutant testes expressed substantially lower levels of pachytene precursor piRNAs.

Interestingly, $A m y b$-mutant testes showed greater downregulation of mature pachytene piRNAs than of precursor pachytene piRNA transcripts, implying that AMYB may have additional roles in piRNA processing. Indeed, they found that AMYB also regulates the expression of piRNA-processing genes, including the pachytene PIWI gene Miwi. Furthermore, AMYB was found to regulate its own expression. Thus, overall, AMYB seems to mediate a robust and coordinated production of pachytene piRNAs. Finally, the authors found similar results in chicken testes, implying that such mechanisms are conserved across vertebrate species.

Unlike in mammals and flies, piRNAs in Caenorhabditis elegans are thought to be mainly transcribed as short transcripts of single piRNA precursors. Each piRNA-encoding locus is characterized by a known, conserved DNA sequence motif $\sim 40$ nucleotides upstream, but the functional features of these cis-elements are poorly characterized. Billi et al. carried out a metaanalysis of small-RNA-sequencing data sets that represent male and female C. elegans germline cells and compared the upstream elements of the piRNA-encoding loci with maleversus female-specific expression. They found that the first nucleotide of the core octamer sequence in the upstream element seems to have a crucial role in sex-specific expression. This was functionally confirmed by engineered alterations to this position, which abrogated the sex bias of expression.

Importantly, the authors showed that simple transgene constructs consisting of an upstream element, a spacer and a piRNA-encoding gene were sufficient to direct the autonomous expression and processing of the piRNA. These engineered piRNAs were expressed at $\sim 10$-fold lower levels than some endogenous clustered piRNA loci, indicating that although these upstream promoters seem to be sufficient to specify piRNA loci and to regulate their transcription, the overall genomic context may also contribute.

Such results add to our emerging understanding of functional features at piRNA-encoding loci.

Darren J. Burgess

ORIGINAL RESEARCH PAPERS Li, X. Z. et al. An ancient transcription factor initiates the burst of piRNA production during early meiosis in mouse testes. Mol. Cell 21 Mar 2013 (doi:10.1016/ j.molcel.2013.02.016) | Billi, A. C. et al. A conserved upstream motif orchestrates autonomous, germline-enriched expression of Caenorhabditis elegans piRNAs. PLoS Genet. 9, e1003392 (2013) 